\title{
Coopération horizontale et gouvernance locale aux Pays-Bas
}

In: Annuaire des collectivités locales. Tome 25, 2005. pp. 171-190.

Citer ce document / Cite this document :

Van Twist Mark J.W., Kort M.B. Coopération horizontale et gouvernance locale aux Pays-Bas. In: Annuaire des collectivités locales. Tome 25, 2005. pp. 171-190.

doi : $10.3406 /$ coloc.2005.1707

http://www.persee.fr/web/revues/home/prescript/article/coloc_0291-4700_2005_num_25_1_1707 


\title{
III. COOPÉRATION HORIZONTALE ET GOUVERNANCE LOCALE AUX PAYS-BAS ${ }^{1}$
}

\author{
M.J.W. VAN TwIST et M.B. KoRT
}

Beaucoup d'études (internationales, comparatives) traitent de et se concentrent sur les relations verticales entre les différents niveaux de l'administration. La présente contribution ne s'intéresse pas à cette distribution verticale. Au lieu de cela, nous nous concentrons sur les relations horizontales (nouvelles et innovantes) qui se font jour actuellement dans le contexte de l'administration publique néerlandaise. Outre celle de l'administration (locale) directement responsable, la participation intense de nombreuses parties publiques et privées constitue la condition la plus importante pour résoudre les problèmes urbains aux Pays-Bas, ou tout au moins réussir à en reprendre le contrôle. Cela met en évidence l'importance d'une capacité organisationnelle bien développée dans l'administration locale. Pour faire suite à cette idée, on a développé aux Pays-Bas des expérimentations avec de nouveaux concepts politiques et des schémas de gouvernance innovants, tels que les «chaînes de gestion » (dans les situations de coopération entre des parties du secteur public), la « démocratie délibérative » (dans les situations de coopération avec la société civile), et les partenariats public-privé (dans les situations de coopération avec des parties du secteur privé).

L'administration locale de Heerlen, une ville du sud des Pays-Bas, doit depuis longtemps faire face à de nombreux problèmes liés aux narcodépendants, aux revendeurs, aux trafiquants de drogue et aux narcotouristes en provenance d'autres pays. L'usage de stupéfiants dans la rue et le trafic de drogue dans des immeubles délabrés

1. Les auteurs remercient D. Puma, M. Noordink et A. Geut pour leur assistance. Article traduit de l'anglais par Véronique Marcou. 
causent de nombreux désagréments à la population de Heerlen : criminalité, troubles à l'ordre public, rassemblements de groupes de consommateurs de stupéfiants, commerce et usage de drogue dans la rue, rixes et prostitution. L'administration locale de Herleen doit traiter le problème en éliminant ce handicap, et sa criminalité corollaire, et en restaurant l'ordre public, sans négliger les intérêts des autres parties impliquées, par exemple les drogués eux-mêmes. L'administration locale est incapable de gérer seule cette affaire, elle cherche donc à développer une coopération avec d'autres parties publiques et privées.

Les Drechtsteden, un partenariat public de collectivités locales, jouissent d'une situation avantageuse sur de grands axes routiers comme l'A15 et l'A16, sur le réseau ferroviaire, et sur les canaux. De plus, les Drechtsteden se trouvent près de l'aéroport et du port de Rotterdam, et disposent d'un complexe industriel très développé dans les secteurs de la construction navale, des infrastructures, et pour la navigation maritime et fluviale. Par ailleurs, le développement de sites industriels prend du retard, on manque d'ouvriers qualifiés, les infrastructures sont tous les jours plus congestionnées et il n'y a aucun établissement d'enseignement supérieur. Tout ceci contribue à donner une image négative des Drechtsteden. La question est la suivante : comment les administrations locales associées peuvent-elles encourager le développement spatial et économique de cette zone ?

Depuis plusieurs années, une zone d'un kilomètre carré autour de la gare centrale d'Utrecht suscite de nombreuses discussions. L'administration locale considère que cette zone - qui n'est pas seulement un carrefour de la circulation des biens et des personnes, mais facilite également la vie, les courses et le travail - risque de se développer de façon anarchique si l'on n'intervient pas par des mesures politiques additionnelles. L'administration locale a donc passé un accord fondé sur une déclaration d'intentions avec les trois plus gros propriétaires immobiliers. Ensemble, ils ont prévu de développer un programme-cadre pour le redéveloppement de la zone. $\mathrm{Ce}$ processus a fait l'objet d'atermoiements successifs suite à plusieurs procédures et au réexamen de décisions antérieures. Les conflits et les crises n'ont pas épargné non plus la coopération mutuelle. Utrecht doit relever le défi consistant à faire avancer ce projet et à éviter des situations comme celle-ci à l'avenir - autant que faire se peut.

Cela n'est qu'un échantillon des problèmes auxquels les administrations locales néerlandaises doivent actuellement faire face. Ces exemples font immédiatement apparaître que ce type de problèmes, lequel tend habituellement à se développer dans les agglomérations les plus vastes, mais pas seulement, présente une configuration diverse et compliquée. Ces problèmes sont mutuellement intriqués, et requièrent la participation de nombreuses parties publiques et privées. De plus, ces différentes parties sont liées par de nombreuses interdépendances. Elles doivent donc travailler ensemble (elles y sont parfois obligées).

La résolution de problèmes sociaux complexes, mais aussi l'incapacité à les traiter, dans un splendide isolement, crée tous les jours de nouveaux défis pour les administrations locales. Les responsables politiques et les fonctionnaires ne doivent pas seulement gérer l'intégralité des problèmes urbains, mais aussi les interactions 
entre les différents porteurs d'intérêts, et la question de savoir quelle forme de coopération est la plus appropriée. De plus, les personnes impliquées ont souvent une vision fondamentalement différente de la définition des problèmes et/ou des solutions envisageables.

On a souvent du mal à obtenir les résultats attendus lorsqu'il s'agit de problèmes urbains. Il est plus ardu encore d'identifier et de mettre à profit toutes les opportunités d'une façon appropriée. On peut en attribuer la cause à certains caractères patents des problèmes urbains ${ }^{2}$ :

- Les problèmes urbains sont fortement intriqués : on ne peut pas les envisager séparément. Ils sont reliés par un réseau de causes et d'effets sous-jacents. Pour prévenir le déclin des relations de voisinage, il ne suffit pas d'entretenir la voirie et de chasser les fauteurs de troubles. Pour éviter que le problème n'apparaisse à d'autres endroits de la ville et que d'autres ne se créent (comme davantage de vols et de vente de stupéfiants dans les cours de lycée), il faut prendre des mesures complémentaires, par exemple, augmenter la qualité des soins.

- Les problèmes urbains peuvent être caractérisés par leurs variations d'échelle : ils surviennent simultanément dans différentes aires politiques et requièrent un investissement coordonné du voisinage, de la ville, de la région, de la province, de l'État et parfois aussi de l'Europe. Même lorsque des problèmes sont clairement attribués à un niveau, par exemple l'administration locale, on ne peut pas y apporter de solution sans l'aide et l'assistance d'administrations d'autres niveaux.

- Les problèmes urbains appellent une approche qui facilite les apports et la participation de plusieurs parties : les apports de riverains et de leurs organisations, d'associations de bénévoles, de développeurs de projets et autres parties privées constituent souvent un prérequis. L'apport des administrations publiques à lui seul ne suffit que de façon anecdotique à apporter une solution adaptée aux problèmes. C'est pourquoi, dans ce contexte, on assiste fréquemment à l'apparition de concepts comme ceux de partenariats public-privé, et de formes participatives et interactives d'élaboration des politiques.

- On peut envisager les problèmes urbains de différents points de vue. En sélectionnant une approche ou une alternative possible pour résoudre les problèmes, chaque point de vue définit ses propres priorités et valeurs, par exemple : la sécurité juridique, l'efficacité et la place faite à la participation. Comme on traite de situations concrètes, cela va s'exprimer par des dilemmes compliqués, comme le choix entre la prudence et la volonté de progresser rapidement, entre réactivité et fiabilité, ou entre solidarité et culture du résultat.

2. WOUDEN, H. VAN DER, De beklemde stad, grootstedelijke problemen in demografisch en sociaaleconomisch perspectief, Rijswijk, SCP, 1996 ; TOPS, P. et WETERINGS, R., Beelden bij « Samenwerken aan leefbaarheid. Over deliberatieve democraties in Breda Noordoost », Tilburg, Stedennetwerk-CRBI, 2001. 
- Les problèmes urbains ont plusieurs objectifs qui peuvent se renforcer les uns les autres, mais aussi entrer mutuellement en conflit. Pour illustrer cette complexité : il est possible d'augmenter la qualité des cadres de vie urbains, par exemple en construisant des maisons réservées à des personnes ayant de hauts revenus. Cependant, de telles politiques ne peuvent réussir que si l'on fait attention à la qualité et à la sécurité de l'environnement, aux infrastructures routières et au niveau de services publics comme l'enseignement et les soins hospitaliers. Investir dans tous ces domaines peut produire des effets d'éviction sur d'autres questions pertinentes, comme la position des résidents actuels sur le marché du travail, ou l'assistance aux personnes socialement vulnérables.

- Les problèmes urbains sont souvent ambigus et leur configuration peut évoluer avec le temps. Les vœux et préférences des parties impliquées peuvent changer, et il ne faut pas non plus sous-estimer les dynamiques de la définition des problèmes. Un jour, les idées au sujet du redéveloppement d'une gare ou l'établissement d'un partenariat urbain peuvent être pertinents et bienvenus, le lendemain considérés comme démodés.

Ces caractéristiques des problèmes urbains sont souvent difficiles et d'un abord hasardeux pour le personnel politique et les fonctionnaires locaux, mais, par ailleurs, elles offrent parfois des chances et des possibilités inattendues ${ }^{3}$. Il est important de prendre conscience que l'environnement urbain ne soulève pas seulement des questions difficiles, il crée également des opportunités par la multiplication des relations et le haut niveau d'interconnexion. Les établissements d'enseignement, les entreprises, leurs clients, les employés, les scientifiques et les artistes sont concentrés dans et autour des villes, et peuvent donc se rencontrer aisément. Le président d'une association de petits commerçants peut rencontrer un élu local, et lancer peu après un projet en partenariat.

Le succès d'une initiative pour résoudre un problème bénéficie parfois à toute la ville. D'autres villes peuvent également bénéficier de ces initiatives. La compétition (croissante) entre les villes les pousse naturellement à rechercher des solutions meilleures que celles de leurs voisines. Des parties qui, autrement, n'auraient jamais été concernées par un problème peuvent s'y trouver impliquées parce que plusieurs objectifs se trouvent mêlés. La confrontation des valeurs et les conflits entre les parties peuvent libérer l'énergie nécessaire pour établir un pont entre elles et parvenir à une solution.

Tous les problèmes urbains et les alternatives prometteuses ont en commun qu'ils dépassent les valeurs et les possibilités des parties individuelles ${ }^{4}$. L'ambition de faire quelque chose pour remédier aux problèmes et utiliser les alternatives pro-

3. Berg, L. VAN DEN, MEer, J. VAN DER et Pol, P., «Organising capacity and social policies in European cities ", Urban Studies, 2003.

4. Burger, Y.D., Veld, R. DS. 'T et Cortlever-Keus, S. (éd.), Facetten van Sioo, Utrecht, Lemma, 2003 ; KLIJN, E.H., Regels en sturing in netwerken : de invlod van netwerkregels op de herstructurering van naoorlogse wijken (thèse), Erasmus Universiteit Rotterdam, 1996. 
metteuses qui se font jour requiert une action collective. En définitive, une action solitaire mènera à une résistance des autres parties impliquées (et de celles intéressées). Quand la politique aboutit à un produit concret (la présentation d'une politique, un plan, une « solution ») et ne prend pas en compte les apports et la dépendance des autres parties, elle ne mènera probablement à aucun résultat satisfaisant. Il est également possible que de nouvelles confrontations apparaissent au cours de la mise en œuvre, et provoquent des retards, ou de nouveaux problèmes.

\section{I) Une approche des problèmes urbains}

Aux Pays-Bas, il existe un consensus sur le fait que les modèles verticaux descendants (qui placent une organisation considérée comme supérieure, verticalement au sommet) sont souvent inadéquats lorsque l'on traite de problèmes urbains ${ }^{5}$. Il n'existe pas aux Pays-Bas un centre d'élaboration de la politique et de pilotage social qui puisse à lui seul imposer une solution. Si nombre d'acteurs ont habituellement la capacité d'éviter à eux seuls d'être contraints à faire quelque chose qu'ils ne veulent pas, ils n'ont généralement pas eux-mêmes de «pouvoir de réalisation ». Compte tenu de la presque inévitable dépendance vis-à-vis des autres acteurs, il est nécessaire de rechercher des partenariats, des coalitions et des alliances. C'est nécessaire aussi parce qu'aucun des acteurs n'est capable d'avoir une vision globale et pertinente des problèmes dans toutes leurs dimensions. Ceux-ci présentent un trop haut degré de complexité, trop de dynamiques sont en jeu. De plus, il est juste de relever que l'innovation (pour des solutions à des problèmes complexes) vient souvent de lieux inattendus, la plupart du temps aux marges des institutions et organisations en place. On recourt de plus en plus à une coopération avec des acteurs multiples pour développer et mettre en œuvre la politique locale.

On ne peut pas demander aux administrations (décentralisées) de développer la zone ferroviaire autour d'Utrecht, de stimuler les activités économiques des Drechtsteden ou d'étendre l'hébergement nocturne pour les narcodépendants d'Utrecht à elles seules. Elles ne disposent pas des savoirs, des compétences et de la capacité humaine nécessaires : il leur manque un pouvoir de réalisation. L'administration doit coopérer avec d'autres acteurs locaux. Les avantages en sont évidents. Donner aux riverains et aux autres acteurs locaux plus de contrôle sur les problèmes locaux dans leur rue et leur voisinage accroît le sentiment de responsabilité vis-à-vis de leur environnement.

5. TEISMAN, G.R., Besluitvorming en Ruimtelijk Procesmanagement. Studie naar eigenschappen van ruimtelijke besluitvorming die realisatie van meervouding ruimtegebruik remmen of bevorderen, Delft, Eburon, 2001. 
On associe au premier abord les termes de coopération et de partenariat avec les notions de chaleur et de compréhension, mais, au quotidien, ces valeurs sont malaisées à appliquer et à maintenir ${ }^{6}$ : on constate des intérêts contradictoires, des responsabilités conflictuelles, l'information est dispersée, les logiques divergent, il y a des différences d'appréhension au sujet de la priorité respective des problèmes, et la vision de l'orientation à suivre change tous les jours. Prendre une décision relativement à des problèmes comme l'ouverture d'un centre d'hébergement pour narcodépendants à un endroit ou à un autre, rendre un centre-ville accessible selon un trajet ou un autre, stimuler les activités économiques en investissant à un certain endroit, et donc pas à un autre : c'est difficile.

Après l'euphorie née de la reconnaissance du besoin de coopérer pour élaborer des documents politiques, des plans, des accords et des visions de développement, on entend des commentaires critiques sur la difficile pratique de la coopération, des alliances et des partenariats. Les parties commencent à se plaindre de discussions sans engagements réels («poldérisation ») et de « séances à la Delarue », où rien ni personne ne progresse. Tout le monde se mêle de tout et, soudain, chacun éprouve le besoin urgent de parler à tout le monde. Les processus s'étirent à l'infini sans aboutir à un progrès concret. La lenteur des processus est l'une des plaintes majeures que l'on entend dans notre pays.

\section{II) Organiser la capacité}

\section{1) Organiser la capacité en tant que concept central}

En matière de problèmes urbains (comme la prévention de la criminalité, la liberté de choisir son enseignement, la désolation sociale, ou les problèmes qui surviennent dans le domaine du renouvellement urbain et de l'amélioration des transports régionaux), on invoque souvent la capacité organisationnelle (ou son absence) pour expliquer le succès ou l'échec dans le contexte de l'administration publique néerlandaise. Si l'on réduit ce phénomène à son essence, il concerne la capacité à résoudre un problème en choisissant les bons porteurs d'intérêts, et à faire converger les opportunités vers un développement durable de la ville ${ }^{7}$.

Quelles sont les formules de coordination et de coopération possibles en matière de capacité organisationnelle et de problèmes urbains ? En règle générale, on a le choix entre :

6. TWIST, M.J.W., Dubbelspel, Publiek-private samenwerking en het management van verwachtingen, Utrecht, Lemma, 2001.

7. Berg, L. Van den, Braun, E. et OtgaAR, A.H.J., Organiserend Vermogen in Perspectief, European Institute for Comparative Urban Research (EURICUR), 2002, p. 2. 
- la coopération entre les administrations locales et les autres organisations gouvernementales : locales, régionales, etc. ;

- la coopération entre les administrations locales et la société civile. Il est possible de définir la société civile comme un ensemble d'individus, d'organisations et d'institutions qu'on ne peut pas considérer comme des parties publiques ou privées déjà impliquées. À cet égard, on peut penser à la coopération avec des personnes civiles, mais aussi avec des mosquées, des fondations privées, etc. ;

- la coopération entre les administrations locales, les sociétés et d'autres institutions à but lucratif (ou plus généralement : des parties privées). On peut formaliser ceci par des accords, des contrats, ou des formules organisationnelles dans lesquelles tous ces types de parties participent.

Dans notre vision, l'intérêt néerlandais pour le concept de « capacité organisationnelle » dans le contexte des problèmes urbains vient de la recherche d'une approche vers une coopération qui ne se perde pas en discussions sans fin. Les gens veulent une approche qui suive le principe « passez aux actes, assez de blabla », une attitude assez répandue actuellement ${ }^{8}$.

En définitive, seule compte la qualité de la mise en ouvre, et pas les plans magnifiques présentant un projet sur le papier. C'est la phase de mise en œuvre que l'administration locale est incapable de mener seule à bien. La grande complainte universelle est « Assez de documents politiques interminables ». Le mot d'ordre devrait être « des résultats, des résultats, des résultats ». Toutefois, il faut prendre conscience qu'une action appropriée est une action coordonnée, conçue pour permettre le dialogue et la coopération avec d'autres parties. On ne doit pas négliger le contexte compliqué dans lequel les problèmes urbains se manifestent, mais plutôt en tirer profit. La demande pour un leadership local ou une action énergique de l'administration publique est une chose, mais ne peut pas trouver de réalisation dans la pratique sans le soutien d'autres parties intelligemment choisies. C'est précisément la situation dans laquelle un concept comme la capacité organisationnelle prend tout son sens ${ }^{9}$. La force de ce concept est qu'il ne s'agit pas seulement d'un autre appel à plus d'efficacité, un nouveau leadership plus puissant et un fort interventionnisme de l'administration publique. La capacité organisationnelle tient compte du contexte complexe et administratif dans lequel les villes doivent opérer. La capacité organisationnelle met l'accent sur la recherche de nouveaux types innovatifs de gouvernance qui rendent productif l'engagement des porteurs d'intérêts.

Dans les paragraphes qui vont suivre, nous allons approfondir l'idée de capacité organisationnelle au moyen de trois concepts politiques à la mode actuellement dans l'administration publique néerlandaise : les chaînes de gestion, la démocratie délibérative et les partenariats public-privé. Chacun d'entre eux décrit une forme spécifique de coopération nécessaire pour une approche effective des problèmes urbains.

8. Voir Zourmis, S. et al., Organiserend vermogen van steden, interne notitie, Tilburg, 2003, p. 1.

9. Ibid. 


\section{2) La coopération entre les parties publiques : les chaînes de gestion}

Les activités liées au service public sont de plus en plus conceptualisées en termes de « chaînes » d'activités ou de parties qui exercent ces activités. Aujourd'hui, l'administration publique néerlandaise est familière de la chaîne de la sécurité, de la chaîne enseignante, de la chaîne du droit d'asile et de la chaîne des soins. Le concept des chaînes de gestion n'est pas uniquement applicable aux infrastructures physiques (par exemple l'eau ou les transports), mais fait également une entrée accélérée dans le champ social, par exemple la délinquance juvénile et l'aide médicale d'urgence.

La société néerlandaise est une société horizontalisée, où la gouvernance peut être assimilée à la négociation. Il en résulte que c'est en fonctionnant que les organisations et administrations locales identifient leurs limites. Ceci explique l'intérêt pour les chaînes de gestion ${ }^{10}$. Les relations de gestion dépassant les limites administratives, juridiques et organisationnelles propres à une institution prennent de plus en plus d'importance. Si l'on cherche à réduire les listes d'attente en matière de soins médicaux ou de suppression des calamités, on "ne peut pas remettre la responsabilité de cette tâche à une seule organisation : les chaînes d'activité jouent un rôle important, et seule une coopération adéquate et mutuelle peut aboutir à un certain niveau de performance et de qualité ${ }^{11}$.

Dans la mise en œuvre des politiques, il est important d'avoir une approche réflexive sur la conception d'une coopération efficace. Les chaînes de gestion se concentrent sur la prestation de services aux consommateurs, et stimulent la connexion des activités entre les organisations publiques et privées. Ces connexions se sont avérées vulnérables : l'optimisation d'un maillon de la chaîne n'améliore pas automatiquement ses performances dans son intégralité ${ }^{12}$. Dans l'évaluation sociale, ce ne sont pas les réalisations individuelles des organisations qui sont déterminantes, mais la façon dont ces performances individuelles se sont inscrites dans un contexte plus vaste et cohérent. Une harmonisation concertée et une communication organisée contribuent à la qualité des services dont jouiront les clients.

Une chaîne est un partenariat entre des parties du secteur public qui sont à la fois indépendantes et mutuellement dépendantes. Elles sont indépendantes, mais ne peuvent pas exister les unes sans les autres. Il y a interdépendance au sens où chaque action d'une partie de la chaîne requiert l'autonomie de l'autre partie. Par exemple : la police ne peut pas combattre la délinquance juvénile sans l'aide de l'assistance à la jeunesse. Le contraire se vérifie également.

10. Dutvenboden, H. van, Twist, M. van, Veldhuizen, M. et Veld, R. DS. 'T (éd.), Ketenmanagement in de publieke sector, Utrecht, Lemma, 2000.

11. AA, A. VAN DER et KONIJN, Th., Ketens, ketenregisseurs en ketenontwikkeling; Het ontwikkelen van transparante samenwerkingsverbanden in netwerken, Utrecht, Lemma, 2001.

12. Duivenboven, H. van et al., Ketenmanagement..., op. cit. 
Le principal objectif d'une chaîne est d'obtenir une certaine harmonisation des activités (et des produits) entre les organisations du secteur public : tous les partenaires de la chaîne ont leurs propres tenants et aboutissants, mais ces derniers ne sont pas tous compatibles. On peut détruire des groupes importants d'étudiants en fin de cycle dans un établissement d'enseignement en leur remettant simplement leur diplôme sans qu'ils soient réellement compétents. Cependant, si ces étudiants poursuivent leurs études ailleurs (à l'université), cela va poser des problèmes. Penser en termes de chaînes (et de chaînes de gestion) contribue au renforcement de l'harmonisation des activités au sein de ce type de connexions.

Le concept des chaînes de gestion encourage les prestataires de services publics à se concentrer sur la demande : cela place les citoyens, en tant que clients, dans une position centrale. Si l'on examine la chaîne à rebours, il apparaît que la prise de conscience de la dépendance mutuelle dans une chaîne convainc les parties de ne plus se concentrer sur leur propre approvisionnement, mais sur la demande des clients et des autres partenaires de la chaîne.

La méthode des chaînes de gestion a été appliquée, dans le contexte néerlandais, à plusieurs cas dans le domaine social, dont des affaires urbaines (mais aussi régionales), liés à des domaines politiques comme la protection infantile, les soins médicaux d'urgence, le droit d'asile, l'enseignement et la Sécurité sociale ${ }^{13}$.

\section{Les chaînes de gestion : résultats de la recherche}

Quels sont les résultats de la recherche néerlandaise sur les chaînes de gestion, relativement à la capacité organisationnelle dans les grandes agglomérations ? Penser en termes de chaînes de gestion a rendu les responsables politiques néerlandais plus attentifs aux risques et goulots d'étranglement suivants, qui peuvent résulter d'une approche standard des affaires urbaines:

- Congestions : parce que la majeure partie des services publics a les caractéristiques d'une chaîne, la capacité d'un maillon peut générer des problèmes non seulement dans sa propre organisation, mais aussi dans d'autres organisations de la chaîne. On pense, par exemple, aux listes d'attente des soins de santé.

- Les effets «waterbed » : des améliorations à un endroit de la chaîne peuvent entraîner des problèmes additionnels à d'autres endroits de la chaîne. Cela arrive dans l'enseignement, quand on baisse le niveau d'exigence aux examens.

- Les fuites: un manque d'attention apporté à l'harmonisation des liens dans la chaîne peut occasionner des pertes indésirables et non intentionnelles. Par exemple, des demandeurs d'asile qui disparaissent pendant la procédure d'enregistrement.

- Les effets "pipeline » : il n'est pas toujours possible d'améliorer la chaîne dans son ensemble. Il arrive que l'effort d'une organisation n'aboutisse qu'à des

13. WOUDEN, H. VAN DER, 2002. 
avantages exclusifs pour une autre organisation de la chaîne. Par exemple, les mesures d'encouragement dans la chaîne du droit pénal.

- Les chaînes croisées : plusieurs chaînes fortement liées se recoupent, rendant difficile d'appliquer une méthode à des groupes cibles. À cet égard, on peut mentionner la méthode des groupes marginaux, dans la politique de la jeunesse.

Dans le secteur public néerlandais, les stratégies destinées à influencer la capacité organisationnelle des chaînes d'administrations locales se fondent sur l'expérience acquise en matière de chaînes de gestion (particulièrement dans le domaine social). On relève les stratégies suivantes :

- Créer une certaine urgence sociale. On peut parfois le faire en attirant l'attention des médias. Cela peut contribuer à structurer des ordres du jour sociaux et politiques, mais ce n'est pas sans risque (polarisation). La recherche peut aussi créer un état d'urgence, et la participation à des plateformes et à des réseaux peut sensibiliser le public concerné.

- Créer une structure commune de référence. On peut citer comme exemple : formuler ses intérêts conjointement, stimuler l'échange des savoirs entre professionnels, créer un fonds d'investissement, activer ses partenaires dans la chaîne, combiner les responsabilités et les flux de capitaux sous un seul dénominateur.

- Stimuler la réactivité, la flexibilité, la variété : rechercher de nouveaux partenaires, connecter les problèmes, utiliser des incitations à l'innovation, par exemple en créant un espace pour l'expérimentation, des zones libres de toutes règles, ou en récompensant les leaders par un espace additionnel pour l'entreprenariat.

- Restructurer les relations à l'intérieur de la chaîne : prêter attention aux incompatibilités entre les incitations dans les diverses chaînes, renforcer la position du client en finançant la demande, nommer un manager pour la chaîne, assigner d'autres rôles (de sélection).

On a aujourd'hui beaucoup d'expérience aux Pays-Bas en matière de chaînes de gestion dans les affaires urbaines. La conclusion selon laquelle les chaînes de gestion, en tant qu'expression d'une coopération (particulièrement) entre des parties du secteur public, ont fait leurs preuves, semble justifiée.

\section{3) La coopération avec la société civile : la démocratie délibérative}

La politique participative, ou interactive, aussi connue sous le nom de démocratie délibérative, est (à côté des chaînes de gestion) un concept à la mode lorsque l'on traite des problèmes (néerlandais) complexes.

La démocratie délibérative est un terme générique qui décrit comment des citoyens, des sociétés, des institutions sociales et autres organisations peuvent contribuer substantiellement au développement et à la réalisation de la politique dans les 
affaires urbaines ${ }^{14}$. La démocratie délibérative a sa propre approche spécifique relativement à la pratique de l'administration urbaine et régionale, et se concentre sur la recherche constante de nouvelles coalitions et alliances entre les parties de la société civile impliquées. Dans la démocratie délibérative, leur participation active est vitale pour le succès de la politique ${ }^{15}$.

Cela fait de la démocratie délibérative un moyen d'organisation de l'engagement collectif. Les riverains, par exemple, peuvent s'impliquer dans les relations de voisinage pour rendre celui-ci plus sûr et plus agréable à vivre, mais par-dessus tout pour exprimer leur sens de la communauté, quels que soient les problèmes en jeu. Dans la démocratie délibérative, les médecins de famille et les employés aux soins de santé ne sont pas uniquement des gens qui soignent (et renvoient les gens à leur travail aussi sec), ils prêtent une oreille attentive et réconfortante ${ }^{16}$.

La démocratie délibérative est souvent fortement associée à la politique interactive et a été considérée comme un mot clé dans le débat néerlandais sur l'administration locale ${ }^{17}$. Elle convient aux Pays-Bas - avec leur tradition de consensus et d'apaisement - comme un gant. Rétrospectivement, on peut être porté à conclure que les idées générées dans les processus de démocratie délibérative peuvent aisément être générées par des professionnels eux-mêmes (ou l'ont déjà été). Toutefois ces idées tirent leur sens et leur statut du fait qu'elles émanent des parties intéressées.

Le développement du concept de démocratie délibérative prend en compte le fait qu'en politique, les parties concernées dans la société civile n'ont souvent que le pouvoir de contrecarrer les ambitions des autres parties - pas celui de réaliser seules les leurs. Quand c'est le cas, le rôle que les parties choisissent de jouer est habituellement un rôle négatif, elles utilisent leur force d'obstruction à défaut d'une alternative plus positive. Souvent, cela mène à une situation où les parties qui font la société civile se développent en obstructionnistes en figures libres, dont le seul but est d'empêcher et de retarder, simplement parce qu'on n'a jamais exigé une approche plus constructive dans le processus.

Affirmer l'existence de ce problème - si important que cela puisse être - ne suffit pas. D'un point de vue prescriptif, quelques questions viennent à l'esprit ${ }^{18}$ : comment éviter des comportement obstructionnistes? comment rendre l'obstruction productive ? comment impliquer les autres parties ? On peut en appeler à toutes les parties concernées pour qu'elles coopèrent, mais la question est de savoir comment faire pour que cela devienne une réalité. La méthode de la démocratie délibérative (ou politique interactive, ou participative) a pour but la formulation de suggestions concrètes pour la conception et la gestion des processus décisionnels. Quelques considérations ${ }^{19}$ :

\footnotetext{
14. TOPS, P., 1994.

15. TEISMAN, G.R., 2004.

16. TOPS, P. et WETERINGS, R., Beelden..., op. cit.

17. Ibid.

18. VAN TwIST, M.J.W., 1999.

19. Teisman, G.R., Complexe besluitvorming : een pluricentisch perspectief op besluitvorming over ruimtelijke investeringen (thèse), 's-Gravenhage, 1991.
} 
- Les chances pour que les acteurs de la société civile acceptent l'issue d'un processus (politique) augmentent sensiblement quand ces acteurs soutiennent le concept de ce processus. Avant le début effectif du processus, les parties de la société civile devraient donc se mettre d'accord sur son fonctionnement.

- Pas d'engagement sans implication. Il est vital d'envisager et de réenvisager sans cesse l'implication de nouveaux acteurs de la société civile, sur la base de leur expertise ou de leur intérêt, même quand la participation de ces parties n'est pas formellement requise.

- On peut obtenir l'implication des acteurs de la société civile au processus en combinant leurs objectifs respectifs, en créant des situations où tout le monde est gagnant, et en créant des offres groupées où chacun trouve son compte. Pour en faciliter la réussite, il faut que la « conception du processus » soit judicieuse.

- Chaque processus politique a ses propres modes de gestion des conflits, comme les accords pour des indemnités (on récupère les profits et on indemnise les pertes, par exemple), ou la validation à l'avance des règles des procédures décisionnelles (par exemple en donnant plus de pouvoir aux minorités qu'aux autres groupes dans les processus décisionnels, ou en accordant un droit de veto à certaines parties). Les parties peuvent aussi s'accorder à l'avance sur des procédures d'arbitrage.

- Accepter l'issue d'un processus ne relève pas que de motifs analytiques et rationnels, mais aussi de motifs sociaux et émotionnels. La conception d'un processus, si elle est judicieuse, tient compte de ces données en laissant aux parties la possibilité de «marquer des points » avec leurs supporters de la base. Ou en lançant de petits projets pilotes ayant de bonnes chances de réussir, et donc de bonnes chances de créer des effets d'entraînement.

- Pour augmenter ses chances d'avoir un impact, il faut organiser un soutien à la base. On peut organiser cela en réglant correctement la participation au processus en termes de compétences et d'intérêts (représentation dirigiste et assistée). Il est également nécessaire de consulter les supporters quand nécessaire : de donner, après coup, un rôle aux parties importantes qui ne peuvent pas participer, et, finalement, de faire ratifier le processus aux parties extérieures au processus.

La mise en application des principes susmentionnés permet d'inciter à davantage d'actions concrètes ${ }^{20}$. L'une d'entre elles est l'activation sélective : on travaille avec certaines parties de la société civile et on en néglige délibérément d'autres. Le choix des parties est une question de contenu mais aussi de processus. Parfois, l'activation d'une partie est nécessaire parce qu'elle possède l'expertise, mais il subsiste toujours le danger que cette partie domine excessivement le processus. Parfois, l'implication d'une partie est nécessaire simplement parce que les lois ou les règlements le requièrent.

20. TERMEER, C.J.A.M., Dynamiek en inertie rondom mestbeleid : een studie naar veranderingsprocessen in het varkenshouderijnetwerk (thèse), 's-Gravenhage, 1993. 
On peut impliquer au processus les parties de la société civile ayant un pouvoir d'obstruction pour qu'il leur soit plus difficile, par la suite, d'en bloquer l'issue, bien que cela n'ait évidemment rien à voir avec le contenu du processus. Souvent, on doit choisir entre donner un pouvoir d'obstruction à une partie au cours du processus lui-même - ralentissant ainsi le processus - et changer légèrement la définition du problème. Parfois on est obligé d'impliquer des parties, même si elles ne peuvent en aucun cas contribuer à un meilleur résultat du processus.

Un processus de conception el de réalisation politique conçu suivant les principes de la démocratie délibérative présente ses propres inconvénients. Au début du processus, les intérêts et priorités des parties impliquées sont habituellement vagues et donc instables. Il n'est pas rare que les intérêts et priorités se dégagent pendant le processus, c'est même souvent lui qui les dessine, par la confrontation avec d'autres parties et leurs objectifs. Certaines parties ne sont capables de définir leur position dans un processus qu'après qu'il a commencé. Cela les pousse à attendre aussi longtemps que possible avant de s'engager définitivement.

Cela rallonge le processus de prise de décision et, en raison de cela, les parties ont tendance à changer légèrement leurs positions (en demandant des délais supplémentaires, ou même en se repositionnant complètement), frustrant ainsi la possibilité d'un aboutissement significatif du processus ${ }^{21}$.

\section{La démocratie délibérative : résultats de la recherche}

Les études néerlandaises sur l'administration locale utilisent la démocratie délibérative pour organiser la capacité des grandes agglomérations à se concentrer en priorité sur la conception des processus. Les conclusions que l'on peut tirer de la démocratie délibérative sont :

- La participation et le militantisme génèrent souvent des idées que les professionnels auraient pu former (ou ont déjà formées) eux-mêmes. Néanmoins, ces idées prennent leur sens et leur statut du fait qu'elles résultent (également) de l'effort des parties de la société civile impliquées. Le changement des procédures standard et des routines des organisations exécutives de façon à incorporer ces nouvelles méthodes de travail constitue une gageure. Dans la pratique, cela génère des frustrations et de l'irritation. À cet égard, une pression externe peut être utile.

- La méthode de la démocratie délibérative ne se concentre pas seulement sur le premier impact (en termes de changements ou d'activités concrètes ou visibles) mais aussi sur le second impact, qui concerne l'implication des citoyens aux problèmes de la zone ou du district où ils vivent. En définitive, le troisième impact de la démocratie délibérative a lui aussi son importance : la façon dont on va stimuler les facultés d'apprentissage des politiciens et de la bureaucratie. Cela se traduit par l'aptitude à réagir à des situations, des opportunités, des contretemps inattendus.

21. Edelenbos, J., Proces in vorm : procesbegeleiding van interactieve beleidsvorming over lokale ruimtelijke projecten (thèse), Utrecht, Lemma BV, 2000. 
- La mise en pratique de la démocratie délibérative est rarement prévisible ou systématique. Cela requiert une attention constante aux ouvertures vers de nouvelles opportunités. La ligne directrice rassemble plusieurs ordres du jour, ce à quoi l'on peut parvenir en anticipant intelligemment les impondérables, les correspondances fortuites, et en maniant l'art de «naviguer à la godille ». L'aptitude à s'adapter et à improviser sont indispensables.

- L'intégration est un mot magique dans l'administration publique. Quand la politique est intégrée, il faut que ce soit de la bonnc politiquc. D'un point de vue pragmatique, l'intégration signifie habituellement: intégré, mais avec une expérience interne à sa propre organisation, prenant part aux délibérations bureaucratiques et adaptant les processus au plus près. Le secret, c'est de faire de l'intégration quelque chose qui ait un sens et soit reconnaissable de l'extérieur : par les citoyens et les autres parties. «Intégré », cela veut dire se concentrer sur le salarié moyen qui ne s'y retrouve pas dans la bureaucratie publique, plutôt que de se reposer sur les chiffres et les statistiques.

- Les capacités du manager du secteur public jouent un rôle de premier plan dans le succès de la démocratie délibérative. Il doit avoir un style personnel combinant professionnalisme, passion, ruse, et être disposé à emprunter des voies peu orthodoxes. De plus, il doit être assisté d'une personne connaissant bien la bureaucratie : un soutien administratif adéquat est indispensable.

- La démocratie délibérative ne consiste pas tant à créer de nouvelles organisations (qui acquièrent une position, des intérêts et du pouvoir en moins de temps qu'il n'en faut pour le dire), mais plutôt à trouver de nouveaux modes d'intervention. On devrait investir dans de nouvelles formes, créatives, de travail, comme la conception d'ateliers ò̀ les parties de la société civile elles-mêmes situent leurs idées de plan urbain sur une carte.

L'objet de la démocratie délibérative, comme des chaînes de gestion, c'est d'accompagner, soutenir et faciliter des processus décisionnels complexes, évaluer les approches spécifiques dont on a besoin pour organiser la capacité des administrations locales. Les chaînes de gestion se concentrent sur les processus primaires de l'administration publique et sur les parties du secteur public dont la contribution est nécessaire pour réaliser un projet. La démocratie délibérative ne se concentre pas sur les processus primaires, ni sur les parties du secteur public dont la participation est obligatoire (ni sur comment les faire coopérer), mais sur la façon dont on peut gérer les pouvoirs d'obstruction et les environnements de réseaux qui sont au cœur des ambitions de la société civile. Ses instruments sont des formes interactives et participatives de développement et de réalisation de la politique.

\section{4) La coopération avec les parties du secteur privé : les partenariats public-privé}

Les partenariats public-privé (PPP) ont fait l'objet d'une grande attention aux Pays-Bas durant les deux dernières décennies. On s'attend à ce que des tendances sociales comme la dérégulation, l'introduction de la concurrence, la libéralisation et les priva- 
tisations augmentent encore l'intérêt pour les PPP dans les années à venir. Les acteurs du secteur privé auront un rôle plus important et davantage de responsabilités dans la conduite et l'initiation de projets faisant intervenir des valeurs relevant du secteur public.

Un PPP est un aménagement organisationnel dans lequel les parties des secteurs public et privé coopèrent à la réalisation d'un projet tout en préservant leurs propres identité et responsabilité. Le partenariat repose sur une répartition claire des tâches et des risques. Dans les PPP, l'administration utilise les capacités commerciales et les capacités d'innovation des parties du secteur privé.

Relativement au développement d'aménagements horizontaux pour l'approche des problèmes urbains, on attend beaucoup des PPP. Quand on s'intéresse de plus près aux documents politiques publiés par différents organismes aux Pays-Bas sur ce sujet, la principale raison de l'adoption de PPP est la valeur ajoutée : un produit de meilleure qualité pour le même investissement financier, ou à un produit de même qualité pour le même prix. La coopération et la combinaison des savoirs et des compétences permettent aux administrations locales de mener à bien leurs projets plus efficacement : c'est une situation où toutes les parties impliquées trouvent leur avantage.

Aux Pays-Bas, on débat actuellement quant à savoir si les aménagements basés sur les contrats de mandat de maîtrise d'ouvrage (principal-agent) (par exemple, les contrats DBFM - Design, Build, Finance and Maintain - et les concessions) peuvent aussi être considérés comme des PPP. Certains pensent que seules les entreprises communes entre des parties des secteurs public et privé (en d'autres termes des partenariats dans le cadre de nouveaux organismes juridiques et organisationnels spécialement créćs) pcuvent être considérées comme des PPP.

Dans la présente contribution, nous nous en tenons au point de départ internationalement admis que les PPP peuvent revêtir des formes diverses. Dans une entreprise commune, on formalise le partenariat en créant un nouvel organisme juridique. Cette organisation est censée réaliser les objectifs mis en commun. Les PPP peuvent également prendre la forme d'un accord qui aboutit ensuite à un contrat entre le principal et l'agent. Lorsqu'on opte pour cette formule, les parties organisent leurs contacts pour discuter des tâches et des responsabilités des différentes parties au partenariat dans la réalisation de leurs objectifs associés. Cette forme de PPP trouve son origine dans la pratique internationale des PPP et dans l'Initiative de financement privé (Private Finance Initiative, PFI).

Jusqu'à une date récente, l'administration centrale néerlandaise s'est concentrée sur les accord contractuels plutôt que sur les entreprises communes. Cela à cause du peu d'expérience que l'on avait aux Pays-Bas de ce second type de formule. Une autre raison en est qu'il est plus difficile de garantir les valeurs du secteur public dans les entreprises communes public-privé. Les intérêts de l'entreprise commune ne sont pas nécessairement les mêmes que les intérêts publics et, d'après la loi, l'administration locale doit se plier aux intérêts de l'entreprise commune. Les intérêts peuvent changer avec le temps. Les règles de l'entreprise commune ne permettent pas un contrôle intégral de l'administration locale parce que ces règles se concentrent sur l'organisation administrative et les divisions hiérarchiques internes. 
Cela veut dire que les valeurs publiques devraient être garanties en premier lieu par la loi et la réglementation, la réglementation des concessions, les contrats, les règles d'attribution, etc. Cela implique que toutes les entreprises communes entre des parties publiques et privées doivent passer par une convention avec la partie du secteur public pour la préservation des valeurs du secteur public. Il est possible que cette convention entre en conflit avec les intérêts des actionnaires.

D'après les théories politiques développées aux Pays-Bas, le second inconvénient des entreprises communes est la relation que les entreprises communes entretiennent avec la réglementation des appels d'offres. Ces réglementations prévoient que s'il advient que les administrations investissent dans une entreprise commune, la participation à cette organisation doit être soumise à un appel d'offres. Les parties du secteur privé ne sont la plupart du temps intéressées par les entreprises communes que si elles voient un profit direct ou indirect à l'investissement. Ce dernier point est difficilement compatible avec l'obligation de soumettre l'entreprise commune aux procédures d'appel d'offres.

Compte tenu des éléments susmentionnés, nous ne pouvons pas conclure simplement que les entreprises communes n'ont pas d'avenir parce qu'elles ne sont pas aptes à réaliser des objectifs sociaux définis. Aux Pays-Bas, on a fait quelques expériences d'entreprises communes. Un exemple récent est la création de ce que l'on a appelé le wijkontwikkelingsmaatschappijen (ou « sociétés pour le développement des zones urbaines ») dans le domaine du développement et de l'aménagement urbains. On crée ces organisations pour réaménager les zones détériorées ou mal développées des agglomérations. Le réaménagement de zones dans le cadre d'un partenariat entre les administrations locales, les organisations de gestion de logements sociaux, des développeurs de projets privés et d'autres parties privées comme les investisseurs institutionnels et les institutions financières s'est avéré une réussite.

D'autre part, aujourd'hui, on recourt aux PPP dans de plus en plus de secteurs. Si les PPP étaient déjà monnaie courante dans le développement de zones urbaines et les infrastructures, ils sont devenus plus fréquents dans des secteurs comme les soins de santé, l'enseignement et les centres pénitentiaires.

De plus, outre l'expérience des PPP que l'on retire de différents projets, l'apprentissage et la diffusion du savoir sont également importants pour le développement de ce type d'aménagements. Récemment, on a réuni, et publié dans des brochures et des manuels les expériences néerlandaises. Entre autres multiples exemples, il y a des manuels sur la gestion du partage des bénéfices, les conventions anti-monopole et les appels d'offres pour les PPP.

\section{Les partenariats public-privé : les résultats de la recherche}

En règle générale, les interactions internes aux projets PPP mettent en évidence des schémas complexes. Les projets de PPP font intervenir de nombreuses parties différentes. Chaque partie (des secteurs public ou privé) a ses propres conceptions et stratégies. On ne peut obtenir un progrès dans la prise de décision que quand ces perceptions et stratégies sont plus ou moins harmonisées les unes avec les autres. De 
plus, dans les projets de PPP, on opère beaucoup de choix qui demandent une prise de décision dans différentes arènes des parties. En règle générale, les PPP sont très ambitieux : la création de valeur ajoutée, plus d'efficacité et des décisions plus rapides. En raison de ces hautes ambitions, les efforts exigés de l'organisation sont également conséquents. La conclusion générale de la recherche sur les PPP est que les interactions sont longues et laborieuses. Dans la pratique néerlandaise, la valeur ajoutée attendue semble difficile à obtenir ${ }^{22}$.

Dans les discussions politiques, la valeur ajoutée est souvent présentée comme un objectif quantitatif à atteindre pour toutes les parties impliquées : de la valeur ajoutée comme objectif, indicateur mesurable pour l'identification d'une situation où chacun trouve son avantage. Cependant, quand l'objectif d'un projet est de faire plus de qualité pour le même prix, ou la même qualité en demandant moins d'argent à l'administration, il est inévitable qu'une ou plusieurs parties du secteur privé fasse un sacrifice. Une administration qui veut construire une autoroute plus sûre à moindre coût va demander un sacrifice aux autres parties impliquées. Un développeur de projet qui a trouvé un moyen innovant de réduire les coûts d'un projet (par exemple : le stockage de l'énergie produite sur la surface des routes, ou la construction d'écoles qui puissent aisément être transformées en maisons dans une nouvelle zone urbaine) n'est pas automatiquement disposé à partager cet avantage avec le gouvernement. En conséquence, de la valeur ajoutée pour une partie ne signifie pas automatiquement qu'il y aura de la valeur ajoutée pour les autres. Au contraire, la valeur ajoutée chez une partie revient souvent à une déperdition de valeur pour l'une des autres parties ou davantagc. Il pcut donc être trompeur de parler de valeur ajoutée comme motif général de choix de PPP (comme nous le faisons aux Pays-Bas), parce que la valeur ajoutée est une notion liée aux intérêts de parties différentes.

Le sentiment que le développement des projets de PPP est insuffisant est répandu aux Pays-Bas. À ce jour, beaucoup ont exprimé leur frustration à ce sujet. Les organisations qui veillent aux interêts des entrepreneurs sont particulièrement promptes à réagir à ce sujet. Les articles parus dans la presse sont éloquents, avec des gros titres comme « Ni la consultation ni les nouveaux documents politiques ne font plus avancer les PPP ». Cet article en particulier affirme : «Si le nombre de mots consacrés au partenariat public-privé au cours des quinze dernières années était normatif pour la mise en œuvre des PPP, on aurait déjà asphalté tous les Pays-Bas à l'heure qu'il est. »

La question est la suivante : comment se fait-il que l'on considère toujours les résultats des PPP comme décevants alors qu'au même moment, presque tout le monde s'empresse d'insister sur les réalisations concrètes : politique, médias, administrations locales et (représentants des) milieux d'affaires ? Il y a sans aucun doute beaucoup d'explications. L'une d'entre elles nous semble pertinente dans ce contexte : dans la pratique des PPP, il y a un tempo paradox.

22. Voir ainsi HAM, J.C. VAN et KOPPENJAN, J., Publiek-private samenwerking bij transportinfrastructuur. Wenkend of wijkend perspectief?, Utrecht, Lemma, 2002. 
In abstracto, tous les partenaires n'ont que des intérêts à une réalisation rapide du projet : les entrepreneurs veulent faire du profit, les échevins [membres de l'exécutif communal] sont confrontés à un environnement politique exigeant qui veut voir des résultats concrets, et à un public impatient qui n'accepte pas les atermoiements tactiques. Cependant, dans la relation mutuelle entre les partenaires sur un projet concret (où on voit surgir des questions comme : qui doit supporter les coûts et prendre les risques ?) laisser paraître son impatience et faire le premier pas peut être une erreur stratégique. Unc fois qu'on a fait des concessions, il est difficile de revenir dessus. D'un point de vue stratégique, il est donc préférable de repousser les concessions autant que faire se peut. Le parti qui a le plus à perdre à un report se trouve dans une mauvaise position pour négocier, en comparaison des autres. Pour progresser, il faut faire des concessions. Le parti qui fait preuve du plus de patience obtient les meilleurs résultats à la fin. Il en résulte que beaucoup, dans le public comme dans le privé, considèrent que la progression des projets PPP est beaucoup trop lente.

\section{5) Des relations verticales aux relations horizontales dans l'administration locale}

La plupart des études sur l'administration locale dans un contexte international se concentrent sur les relations verticales entre différents niveaux administratifs. Dans la présente contribution, nous avons choisi de ne pas nous concentrer sur ce type dc rclations. Au lieu de cela, nous avons choisi de nous concentrer sur les relations horizontales qui se font jour actuellement dans l'administration locale néerlandaise.

Nous avons exploré de nouveaux concepts pour comprendre et réformer ces relations. Dans le tableau suivant, nous présentons le contexte et les grandes lignes de ces concepts.

\section{Tableau I}

\begin{tabular}{|l|l|}
\hline Concept & $\begin{array}{l}\text { Construction destinée à organiser la capacité relativement aux pro- } \\
\text { blèmes urbains. }\end{array}$ \\
\hline Chaînes de gestion & $\begin{array}{l}\text { Mode d'organisation des tâches relatives aux problèmes urbains, et } \\
\text { où la coopération concerne les services publics et vise à la réalisation } \\
\text { d'un partenariat plus axé sur la demande entre les parties publiques. }\end{array}$ \\
\hline $\begin{array}{l}\text { Démocratie } \\
\text { délibérative }\end{array}$ & $\begin{array}{l}\text { Un style de gouvernance adapté à la tradition néerlandaise de consul- } \\
\text { tation et de consensus. Ce style ne vise pas seulement la résolution } \\
\text { instrumentale de problèmes, mais aussi l'implication de la société } \\
\text { civile. }\end{array}$ \\
\hline $\begin{array}{l}\text { Les partenariats } \\
\text { public-privé }\end{array}$ & $\begin{array}{l}\text { Dans les PPP, l'administration utilise les capacités commerciales et } \\
\text { les capacités d'innovation du secteur privé. Les partenariats sont for- } \\
\text { malisés par une entreprise commune ou un accord contractuel. }\end{array}$ \\
\hline
\end{tabular}




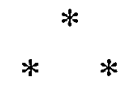

En conclusion à cette contribution, nous dirons que l'on part fréquemment du présupposé que, pour une approche efficace de la résolution des problèmes dans la pratique locale, une implication intensive des administrations est un prérequis. On peut remettre en cause cette affirmation. Après un examen attentif, on peut se demander si, pour une approche efficace des problèmes urbains, l'implication directe ou indirecte des administrations locales est toujours nécessaire. Si cela se vérifiait, cela voudrait dire que l'administration est toujours la partie qui fait la différence (et que l'implication des autres parties n'en fait aucune).

- Au lieu de cela, nous partons de l'idée qu'il devrait y avoir au départ plus de coopération et de partenariat entre l'administration locale et d'autres organisations (de la société civile publique et privée). On devrait prêter plus d'attention aux initiatives pour la coopération et les partenariats entre ces autres organisations sans qu'une administration locale y soit directement impliquée. Par exemple, les acteurs du secteur privé (les sociétés) et les organisations qui font partie de la société civile. L'intérêt porté à ces organisations à buts lucratif et non lucratif dans le cadre d'une approche de la base vers le haut compense l'image bureaucratique de modèle de communication descendant qui prévaut, dans les représentations de la réalité et les intérêts des institutions administratives qui dominent encore parfois, aujourd'hui, le débat politique sur la façon de traiter les problèmes urbains.

Les organisations de gestion de logements sociaux qui, dans le cadre de PPP avec les cliniques, s'efforcent de réduire les listes d'attente dans le domaine des soins de santé constituent un exemple de cette approche de la base vers le haut. On peut citer un autre exemple, concernant les chaînes de gestion, où une institution pour les sans-abri a décidé de proposer des locaux pour étudier, en coopération avec une institution d'enseignement régionale. Enfin, on peut mentionner une ferme-école qui ne se contente plus de ses activités agraires, mais organise également des soins de jour pour les handicapés, suite à une décision prise dans le cadre de la démocratie délibérative.

Les politiques d'administration locale sont rarement à l'origine des innovations mentionnées plus haut. L'attention, au niveau de la politique locale, se tourne majoritairement vers le pôle opposé (en raison d'incidents d'ordre plus ou moins important) : la réglementation et la comptabilité, la planification et le contrôle, les conditions et les procédures, censées tout prendre en compte, tout contrôler et ne rien laisser passer (mieux que par le passé).

Il va de soi que les administrations ont une part aux processus quand on pense aux chaînes de gestion, à la démocratie délibérative et aux PPP. Mais ce n'est plus toujours le point de départ central lorsqu'il s'agit de traiter des problèmes urbains locaux. Dans le contexte néerlandais contemporain, il n'est pas rare qu'en fait, elles suivent les actions et les initiatives d'autres parties (de la société civile publique et privée). 


\section{Bibliographie}

BRUIJ, J.A. DE et Heuvelhof, E.F. TEN, Management in Netwerken, Utrecht, Lemma, 1999.

BRuiJ, J.A. DE, Heuvelhof, E.F. TEN et Veld, R.J. DS. 'T, Procesmanagement : Over procesontwerp en besluitvorming, Schoonhoven, Academic Service, 1998.

Commissie CerfontaINE, ICT en de stad : Burgers verbonden, Den Haag, Sdu, 2000.

Edelenbos, J., Reudink, M. et HoORn, T. van, Beelden en verwachtingen over de Zwolse Variant, Tussenrapportage naar aanleiding van de nulpuntmeting in het evaluatieonderzok 'de Zwolse Variant: Wijkgericht Werken in Zwolle, Rotterdam/Delft, TNO-rapport, 2001.

EDELENBOS, J. et MONNIKHOF, R. (redactie), Lokale interactieve beleidsvorming. Een vergelijkend onderzok naar de consequenties van interactieve beleidsvorming voor het functioneren van de lokale democratie, Utrecht, Lemma, 2001.

HEUVELHOF, E.F. TEN, Binnengrenzen Haaglanden, Extern rapport in opdracht van Stadsgewest Haaglanden, 1996.

Hendriks, F. et Toonen, Th. A.J. (éd.), Schikken en plooien, De stroperige staat bij nader inzien, Van Gorcum, Assen, 1998.

SocIAAl ECONOMISCHE RAAD, Samen voor de stad, 1998.

TEISMAN, G.R., Complexe besluitvorming, een pluricentrisch perspectief op besluitvorming over ruimtelijke investeringen, 's-Gravenhage, 1995.

TEISMAN, G.R. et VELD, R. DS. 'T (éd.), Over effectieve structuren tussen overheid en bedrijfsleven - Mogelijkheden van en voorstellen tot een gemeenschappelijke publiek-private aanpak van maatschappelijke problemen, 's-Gravenhage, 1992.

Twist, M.J.W. et VeEneman, W.M., Marktwerking op weg : over concurentiebevordering in infrastructuurgebonden sectoren, Utrecht, Lemma, 2002.

ZouridIs, S., De regionale horde in het Grotestedenbeleid, Tilburg, ds. opdracht van het Kenniscentrum Grote Steden, 2003. 